\title{
Adverse events in users of sertraline: results from an observational study in psychiatric practice in The Netherlands
}

\author{
Welmoed E. E. Meijer MSc*1,2, Eibert R. Heerdink $\mathrm{PhD}^{1}$, Jacques ThM. van Eijk MD, PhD ${ }^{3}$ \\ and Hubert G. M. Leufkens $\mathrm{PhD}^{1}$ \\ ${ }^{1}$ Department of Pharmacoepidemiology and Pharmacotherapy, Utrecht Institute for Pharmaceutical Sciences, Utrecht, \\ The Netherlands \\ ${ }^{2}$ Kendle International, Utrecht, The Netherlands \\ ${ }^{3}$ Department of Medical Sociology, Health Care Studies, Faculty of Medicine, University of Maastricht, The Netherlands
}

\section{SUMMARY}

Purpose To evaluate the safety profile of sertraline versus other Selective Serotonin Reuptake Inhibitors (SSRIs) directly following the introduction of sertraline to the Dutch market.

Methods In a prospective follow-up study, 109 psychiatrists included patients with a new episode of treatment with sertraline and an equal number of patients starting treatment with other SSRIs. All Adverse Events (AEs) during follow-up were recorded by the psychiatrists for the duration of SSRI treatment until discontinuation or until at least 12 months.

Results A total of 1251 patients were included in the study of which 659 used sertraline and 592 used other SSRIs (paroxetine, fluoxetine or fluvoxamine). The most frequently reported events in sertraline users and users of other SSRIs were nausea $(160(24.3 \%)$ sertraline patients versus 160 (27.0\%) patients using other SSRIs), headache (127 (19.3\%) sertraline patients versus $101(17.1 \%)$ patients using other SSRIs), diarrhoea (94 (14.0\%) sertraline patients versus 40 patients using other SSRIs $(6.8 \%, p<0.05))$, sweating $(88(13.4 \%)$ sertraline patients versus $69(11.7 \%)$ patients using other SSRIs) and dizziness $(75$ (11.4\%) sertraline patients versus 70 (11.8\%) patients using other SSRIs). A total of 121 patients reported 134 different unlabelled AEs of which 10 were reported by more than $1 \%$ of the population.

Conclusions In this study we found that almost three out of four patients reported an adverse event. When comparing with other SSRIs and the literature, we found a similar distribution of the most frequently reported adverse events in patients using sertraline. However, in this observational study we found over 100 different unlabelled adverse events. Copyright (C) 2002 John Wiley \& Sons, Ltd.

KEY WORDS — sertraline; adverse events; SSRIs; pharmacoepidemiology

\section{INTRODUCTION}

Monitoring the safety of a new drug directly following its introduction into clinical practice is important in order to detect unknown or rare adverse events

\footnotetext{
* Correspondence to: W. E. E. Meijer, Department of Pharmacoepidemiology and Pharmacotherapy, Utrecht Institute for Pharmaceutical Sciences (UIPS), PO Box 80.082, 3508 TB Utrecht, The Netherlands. Tel: +31 30 2537324. Fax: +31302539166.

Contract/grant sponsor: Pfizer bv.
}

Copyright (C) 2002 John Wiley \& Sons, Ltd.
(AEs) that have not previously been seen in preregistration trials. ${ }^{1}$ Various study designs can be used to study safety issues, including randomized controlled trials (RCTs) after registration comparing the new drug with other/older drugs, case reports, spontaneously reported events and observational cohort studies. Possible limitations of these designs are that the controlled circumstances of the RCT are not comparable with the situation in daily practice, and that case reports/spontaneous reports stem from undefined populations in which data is collected in a non-standardized manner. ${ }^{2,3}$ Studies representative 
for the general population provide more information about the population at risk and put spontaneous reports in perspective.

Safety of selective serotonin reuptake inhibitors (SSRIs) in clinical practice should be evaluated in the context of usage patterns and the dynamics of the prescribing of new drugs in often overcrowded markets of existing compounds with similar therapeutic characteristics. Egberts et al. have reported that newly introduced antidepressant drugs (e.g. sertraline, mirtazapine and venlafaxine) were more often prescribed by psychiatrists to patients with prior prescriptions of another antidepressant or other psychotropic drugs ('channelling'). ${ }^{4}$ This phenomenon was previously shown by Petri et al. with the antidepressant mianserin. ${ }^{5}$ Selective prescribing of certain drugs in populations with different baseline risks when compared with a group of atypical patients, begs the question 'did the drug bring the problems to the patient, or did the patient bring the problems to the drug?' in cases where a safety issue emerges. ${ }^{6}$

This was the reason for setting up an observational, prospective study directly following the introduction of sertraline into psychiatric practice in The Netherlands. At the time of the introduction of sertraline in 1994, three other (fluvoxamine, fluoxetine and paroxetine) SSRIs were available in The Netherlands. The objective of this study was to evaluate the pattern of adverse events (AEs) in users of sertraline compared to users of other SSRIs which have been available for longer.

\section{PATIENTS AND METHODS}

This prospective, observational follow-up study was conducted in The Netherlands from 1 January 1995 until 31 December 1997. A total of 1251 patients were followed for 12 months or until they discontinued therapy. The study protocol was approved by the Medical Ethics Committee of the University Medical Centre, Utrecht. This study was designed according to the Guidelines for Safety Assessment of Marketed Medicines (SAMM). ${ }^{7}$

A sample of 559 psychiatrists were asked to participate in the study and of these a total of 109 agreed to participate in the study. They were located in psychiatric $(16.5 \%)$ and general hospitals $(21.1 \%)$, regional institutes of mental health $(39.4 \%)$ or in private practices $(22.9 \%)$. The majority of the psychiatrists were male $(85 \%)$. Psychiatrists recorded all prescriptions in a prescription log and asked all patients with a new sertraline prescription to participate in the study. Consecutive patients using fluoxetine, fluvoxamine or paroxetine were asked to be control patients. No additional inclusion or exclusion criteria were applied. Written informed consent was obtained from all patients that were included.

Information on patient characteristics (age and gender), diagnosis and comorbidity at the inclusion visit were recorded by the psychiatrist for both treatment groups in an identical manner. At each visit the patient was asked by open questions (i.e. not by checklist) for all medical AEs irrespective of a possible association with SSRI use. The relationship with the use of an SSRI was classified by the physician as 'unlikely', 'possible' or 'probable' for all reported events. ${ }^{8}$ Start and stop dates of the events and assessment of severity ('mild', 'moderate' or 'severe') were recorded. All adverse events were coded by the authors according to the adverse reaction terminology of the WHO and unclear events were coded with the assistance of a qualified assessor from The Netherlands Pharmacovigilance Foundation. Unlabelled AEs were defined as events not listed in the Summary of Product Characteristics (SPC) of sertraline or other SSRIs. ${ }^{9}$ In a comparison with data from the UK prescription event monitoring (PEM) system we adjusted for differences between our coding list and the PEM classification by adding the individual categories of headache/migraine, agitation/panic attacks and nausea/vomiting.

Serious adverse events (SAEs) were recorded according to the guidelines in the International Conference on Harmonization of Good Clinical Practice (ICH-GCP). ${ }^{10}$ An event that resulted in death, was life threatening, required hospitalization or prolongation of the existing hospitalization, resulted in persistent or significant disability or incapacity or produced a congenital anomaly or birth defect was recorded as an SAE. Psychiatrists were asked to report any SAE within $24 \mathrm{~h}$ after the event. As well as the description of the event (with start date and stop date), the association with the use of the SSRI and the action taken ('dose changes', 'comedication' or 'discontinuation of the SSRI') were also recorded.

We corrected for possible confounders by including sex, age, indication and psychiatric comorbidity in a Poisson regression model. We compared our results with the results of a large observational cohort study in the UK using PEM data. ${ }^{11}$ Proportions of reported AEs compared to the total number of events reported were calculated for our study and for the PEM study using figures given in the supplement of the study by Freemantle et al. $^{12}$

Results were tabulated in absolute values and percentages. Statistical significance was calculated with the $\chi^{2}$ test, with $p<0.05$ as the criterion for significance. 


\section{RESULTS}

A total of 1251 patients were included: 659 patients using sertraline and 592 patients using other SSRIs. Characteristics of all patients are shown in Table 1. The majority of the patients were female and the median age was 41 years (range 6-95 years). The main indication for prescribing an SSRI was depressive disorder $(77.9 \%)$, followed by anxiety disorder $(15.5 \%)$. A total of 473 patients $(37.8 \%)$ had multiple diagnoses of which depressive disorder combined with anxiety disorder and depressive disorder combined with personality disorder were most frequently seen. Previous use of antidepressants was recorded in $44.8 \%$ of the patients. Patients were followed for an average duration of 5.7 months (range 1-365 days).

No differences were seen between users of sertraline compared to users of other SSRIs with respect to sex, age and comorbidity either former or current. The majority of the patients $(n=975,77.9 \%)$ had the indication depressive disorder which occurred significantly more often among patients using sertraline $(n=546,82.9 \%)$ as compared to those using other SSRIs $(n=429,72.5 \%)$. Anxiety disorder was seen significantly less frequently in sertraline patients $(n=74,11.2 \%)$ compared to patients using other SSRIs $(n=120,20.3 \%)$. Also multiple diagnoses, including depressive disorder and anxiety disorder, were significantly less frequently seen in patients using sertraline compared to patients using other SSRIs (51 patients $(7.7 \%)$ vs. 66 patients $(11.1 \%)$ ). The combination of depressive disorder and personality disorder was seen significantly more frequently in patients using sertraline (69 patients, $10.5 \%$ vs. 39 patients $6.6 \%$ ). The average follow-up duration for all patients was 172.9 days (range 1-690 days); patients using sertraline had an average follow-up duration of 173.8 days (range 1-612 days) compared to patients using other SSRIs who had an average follow-up duration of 171.8 days (range 2-690 days). No major differences in average follow-up duration between users reporting adverse events using sertraline (175.8 days, range 1-612 days) and users of other SSRIs (171.9 days, range 2-601 days) were seen.

In total, 928 patients $(74.1 \%)$ reported an $\mathrm{AE}$, including $484(73.4 \%)$ patients using sertraline and $444(75.0 \%)$ patients using other SSRIs. On average 2.2 AEs per patient were reported (2.2 AEs in users of sertraline vs. 2.1 AEs in patients using other SSRIs). One AE was reported by 234 patients (18.7\%), two AEs by 223 patients (17.8\%), three AEs by 180 patients (14.4\%), four AEs by 130 patients (10.4\%) and more than five AEs by 165 patients $(13.2 \%)$. A total of 2713 AEs were reported

Table 1. Patient characteristics

\begin{tabular}{|c|c|c|c|c|}
\hline & $\begin{array}{c}\text { All patients } \\
n=1251(\%)\end{array}$ & $\begin{array}{c}\text { Sertraline } \\
n=659(\%)\end{array}$ & $\begin{array}{l}\text { Other SSRIs } \\
n=592(\%)\end{array}$ & $p$-value \\
\hline Males & 449 (35.9) & $244(37.0)$ & 205 (34.6) & 0.3776 \\
\hline \multicolumn{5}{|l|}{ Age (years) } \\
\hline$<18$ & $19(1.5)$ & $8(1.2)$ & $11(1.9)$ & 0.3523 \\
\hline $18-44$ & $720(57.6)$ & $380(57.7)$ & $340(57.4)$ & 0.3581 \\
\hline $45-64$ & $322(25.7)$ & $178(27.0)$ & $144(24.3)$ & 0.2631 \\
\hline$>=65$ & $190(15.2)$ & $93(14.1)$ & $97(16.4)$ & 0.5703 \\
\hline \multicolumn{5}{|l|}{ Somatic history of } \\
\hline Heart disease & $65(5.2)$ & $33(5.0)$ & $32(5.4)$ & 0.3802 \\
\hline Diaetes & $26(2.1)$ & $17(2.6)$ & $9(1.5)$ & 0.1897 \\
\hline \multicolumn{5}{|l|}{ Psychiatric history of } \\
\hline Depressive disorder & $35(2.8)$ & $15(2.3)$ & $20(3.4)$ & 0.2381 \\
\hline Other psychiatric disorder & $70(5.6)$ & $34(5.2)$ & $36(6.1)$ & 0.4790 \\
\hline Social problems & $14(1.1)$ & $10(1.5)$ & $4(0.7)$ & 0.1576 \\
\hline \multicolumn{5}{|l|}{ Indication } \\
\hline Depressive disorder & 975 (77.9) & $546(82.9)$ & $429(72.5)$ & $<0.001$ \\
\hline Anxiety disorder & $194(15.5)$ & $74(11.2)$ & $120(20.3)$ & $<0.001$ \\
\hline Other & $82(6.6)$ & $39(5.9)$ & $43(7.2)$ & 0.3372 \\
\hline \multicolumn{5}{|l|}{ Psychiatric comorbidity } \\
\hline Depressive and anxiety disorder & $117(9.4)$ & $51(7.7)$ & $66(11.1)$ & 0.0387 \\
\hline Depressive and personality disorder & $108(8.6)$ & $69(10.5)$ & $39(6.6)$ & 0.0147 \\
\hline \multicolumn{5}{|l|}{ Previous use of } \\
\hline TCA & $166(13.3)$ & $94(14.3)$ & $72(12.2)$ & 0.2741 \\
\hline SSRI & $226(18.1)$ & $128(19.4)$ & 98 (16.6) & 0.1880 \\
\hline Benzodiazepines & $221(17.7)$ & $109(16.5)$ & $112(18.9)$ & 0.2709 \\
\hline Antipsychotics & $71(5.7)$ & $36(5.5)$ & $35(5.9)$ & 0.7317 \\
\hline
\end{tabular}


Table 2. Adverse events in all patients: severity, association with use of SSRI and action taken by prescriber

\begin{tabular}{lccc}
\hline & Total & Sertraline & Other SSRIs \\
& $2713(\%)$ & $1459(\%)$ & $1254(\%)$ \\
\hline Severity & & & \\
$\quad$ Mild & $970(35.8)$ & $518(35.5)$ & $452(36.0)$ \\
Moderate & $1088(40.1)$ & $574(39.3)$ & $514(41.0)$ \\
Severe & $640(23.6)$ & $358(24.5)$ & $282(22.5)$ \\
$\quad$ Unknown & $15(0.6)$ & $9(0.6)$ & $6(0.5)$ \\
Association with & & & \\
use of SSRI & & & $123(9.8)$ \\
Unlikely & $290(10.7)$ & $167(11.4)$ & $363(28.9)$ \\
Possibly & $795(29.3)$ & $432(29.6)$ & $764(60.9)$ \\
Probably & $1612(59.4)$ & $848(58.1)$ & $4(0.3)$ \\
Unknown & $16(0.6)$ & $12(0.8)$ & \\
\hline
\end{tabular}

during the study period: 1459 AEs among users of sertraline and 1254 AEs among users of other SSRIs (Table 2). The majority of AEs were labelled as 'moderate' $(n=1088,40.1 \%)$ or 'mild' $(n=970,35.8 \%)$ by the physician. Most AEs were categorized as probably associated with use of the SSRI, with no differences between sertraline and the other SSRIs.
The most frequently reported AEs (Table 3) were nausea in 160 sertraline patients $(24.3 \%)$ vs. 160 patients $(27.0 \%)$ using other SSRIs and headache in 127 patients (19.3\%) using sertraline vs. 101 patients $(17.1 \%)$ using other SSRIs. After adjustment for possible confounders in a Poisson regression model, diarrhoea (adjusted Incidence Rate Ratio (95\% confidence interval): 1.87 (1.28-2.73)) and abdominal pain (IRR (95\% CI): 2.30 (1.18-4.49)) were significantly more frequently reported by users of sertraline compared to users of other SSRIs. No significant differences were found between patients using sertraline and patients using other SSRIs in the incidences of the other most frequently reported AEs including insomnia, dizziness, agitation, loss of concentration, tremor, anxiety, visual disturbances, palpitations, nausea, sexual dysfunction, sweating and dry mouth.

A total of 57 patients $(4.6 \%)$ reported 59 serious adverse events (SAEs) as defined by the ICH-GCP guidelines, including two patients with multiple (2) SAEs. No significant differences in SAE reporting were found between patients using sertraline $(n=33$, $5.0 \%$ ) compared to patients using other SSRIs

Table 3. Incidence (per 1000 person-years) of adverse events (AEs) reported by patients using sertraline compared with patients using other SSRIs

\begin{tabular}{|c|c|c|c|}
\hline & $\begin{array}{c}\text { Sertraline* } \\
n \text { (incidence) }\end{array}$ & $\begin{array}{l}\text { Other SSRIs* } \\
n \text { (incidence) }\end{array}$ & $\begin{array}{l}\text { Incidence Rate Ratio } \\
\text { (95\% confidence interval) }\end{array}$ \\
\hline \multicolumn{4}{|l|}{ CNS/Psychiatric } \\
\hline Headache & $127(1.11)$ & $101(0.99)$ & $1.07(0.82-1.40)$ \\
\hline Insomnia & $53(0.46)$ & $35(0.34)$ & $1.33(0.86-2.05)$ \\
\hline Dizziness & $75(0.65)$ & $70(0.69)$ & $0.95(0.68-1.32)$ \\
\hline Agitation (including panic attacks) & $51(0.45)$ & $31(0.30)$ & $1.54(0.98-2.43)$ \\
\hline Loss of concentration & $36(0.31)$ & $45(0.44)$ & $0.66(0.42-1.03)$ \\
\hline Tremor & $51(0.45)$ & $47(0.46)$ & $0.98(0.66-1.47)$ \\
\hline Anxiety & $20(0.17)$ & $13(0.13)$ & $1.48(0.72-3.03)$ \\
\hline Visual disturbances & $20(0.17)$ & $21(0.21)$ & $0.89(0.48-1.67)$ \\
\hline \multicolumn{4}{|l|}{ Cardiovascular } \\
\hline Palpitations & $21(0.18)$ & $13(0.13)$ & $1.39(0.69-2.82)$ \\
\hline \multicolumn{4}{|l|}{ Gastrointestinal } \\
\hline Nausea & $160(1.4)$ & $160(1.57)$ & $0.91(0.72-1.14)$ \\
\hline Diarrhoea & $92(0.8)$ & $40(0.39)$ & $1.87(1.28-2.73)$ \\
\hline Abdominal pain & $12(0.1)$ & $34(0.33)$ & $2.30(1.18-4.49)$ \\
\hline \multicolumn{4}{|l|}{ Sexual dysfunction } \\
\hline Loss of libido & $31(0.27)$ & $19(0.19)$ & $1.52(0.85-2.71)$ \\
\hline Ejaculation failure & $14(0.12)$ & $22(0.22)$ & $0.56(0.28-1.10)$ \\
\hline Impotence (male) & $9(0.08)$ & $9(0.09)$ & $0.76(0.30-1.92)$ \\
\hline Other sexual function disorder (male) & $4(0.03)$ & $3(0.03)$ & $1.35(0.36-5.14)$ \\
\hline Anorgasmia (female) & $6(0.05)$ & $8(0.08)$ & $0.66(0.22-1.93)$ \\
\hline Other sexual function disorder (female) & $3(0.03)$ & $4(0.04)$ & $1.00(0.30-3.30)$ \\
\hline \multicolumn{4}{|l|}{ Autonomic } \\
\hline Sweating & $88(0.77)$ & $69(0.68)$ & $1.13(0.82-1.56)$ \\
\hline Dry mouth & $46(0.40)$ & $46(0.45)$ & $0.84(0.55-1.27)$ \\
\hline
\end{tabular}

*Incidence is the number of first adverse events/1000 person-years. Total person time at risk for sertraline users was 114563 years versus users of other SSRIs 101734 years.

${ }^{\dagger}$ Adjusted for sex, age, indication and psychiatric comorbidity by Poisson regression analysis. 
$(n=26,4.6 \%)$. All SAEs resulted in hospitalization in either a psychiatric hospital or psychiatric ward (36 SAEs, $61.0 \%$ ) or a general hospital (23 SAEs, $39.0 \%)$. A total of three patients $(0.2 \%)$ died after hospitalization but no relationship with SSRI use was reported by the prescriber. Psychiatric SAEs included suicide attempts (five patients using sertraline $(0.8 \%)$ versus 10 patients $(1.7 \%)$ using other SSRIs) or worsening of depressive status (12 patients $(1.8 \%)$ using sertraline versus seven patients (1.2\%) using other SSRIs). During follow-up, two patients were admitted to a psychiatric hospital twice (one patient using sertraline versus one patient using paroxetine) due to worsening of the depressive symptoms. Somatic SAEs were reported including dizziness, hyponatremia, joint inflammation, thrombosis, fall, death, hypoglycaemia, loss of memory, broken leg (two patients), carcinoma (three patients), cardiovascular reasons (four patients) and gastrointestinal reasons (six patients). In one patient, using paroxetine, the admission (reason: hyponatremia) was related to the use of the SSRI, in all

Table 4. Proportion of adverse events to total number of adverse events reported in users of sertraline

\begin{tabular}{|c|c|c|}
\hline & $\begin{array}{c}\text { Sertraline } \\
\text { (our results) }\end{array}$ & $\begin{array}{c}\text { Sertraline } \\
(\text { Freemantle } \text { et al. })^{12}\end{array}$ \\
\hline $\begin{array}{l}\text { Total number of } \\
\text { events reported } \\
\text { CNS }\end{array}$ & 1459 & 4814 \\
\hline $\begin{array}{l}\text { CNS } \\
\text { Headache/migraine* }\end{array}$ & CNS & $343(7.1)$ \\
\hline Insomnia & $56(3.8)$ & $196(4.1)$ \\
\hline Dizziness & $77(5.3)$ & 177 (3.7) \\
\hline Agitation/panic attacks* & $57(3.9)$ & $157(3.3)$ \\
\hline Tremor & $51(3.5)$ & $110(2.3)$ \\
\hline Anxiety & $22(1.5)$ & $68(1.4)$ \\
\hline Visual disturbances & $21(1.4)$ & $24(0.5)$ \\
\hline \multicolumn{3}{|l|}{ Cardiovascular } \\
\hline Palpitation & $22(1.5)$ & $47(1.0)$ \\
\hline \multicolumn{3}{|l|}{ Gastrointestinal } \\
\hline Nausea/vomiting* & $191(13.1)$ & $540(11.2)$ \\
\hline Diarrhoea & $101(6.9)$ & $347(7.2)$ \\
\hline Abdominal pain & $35(2.4)$ & $187(3.9)$ \\
\hline \multicolumn{3}{|l|}{ Sexual dysfunction } \\
\hline Loss of libido & $31(2.1)$ & $\dagger$ \\
\hline Ejaculation failure & $14(1.0)$ & $21(0.4)^{\ddagger}$ \\
\hline Impotence (male) & $11(0.8)$ & $\dagger$ \\
\hline $\begin{array}{l}\text { Other sexual function } \\
\text { disorder (male) }\end{array}$ & $5(0.3)$ & $\dagger$ \\
\hline Anorgasmia (female) & $6(0.4)$ & $\dagger$ \\
\hline $\begin{array}{l}\text { Other sexual function } \\
\text { disorder (female) }\end{array}$ & $6(0.4)$ & $\dagger$ \\
\hline \multicolumn{3}{|l|}{ Autonomic } \\
\hline Sweating & $91(6.2)$ & $83(1.7)$ \\
\hline Dry mouth & $47(3.2)$ & $47(1.0)$ \\
\hline
\end{tabular}

*Combination of categories due to differences in coding dictionaries. ${ }^{\dagger}$ No specific information available.

${ }_{\ddagger}^{\ddagger}$ Impotence, ejaculation failure.

Copyright (C) 2002 John Wiley \& Sons, Ltd.
Table 5. Unlabelled adverse events reported by at least $1 \%$ of all patients

\begin{tabular}{lcr}
\hline Adverse event & $\begin{array}{c}\text { Sertraline } \\
n=659(\%)\end{array}$ & $\begin{array}{r}\text { Other SSRIs } \\
n=592(\%)\end{array}$ \\
\hline Dyspepsia & $28(4.2)$ & $25(4.2)$ \\
Appetite increased & $11(1.7)$ & $7(1.2)$ \\
Yawning & $10(1.5)$ & $10(1.7)$ \\
Paraesthesia & $10(1.5)$ & $3(0.5)$ \\
Dreaming abnormal & $9(1.4)$ & $12(2.0)$ \\
Personality disorder (attitude change) & $9(1.4)$ & $10(1.7)$ \\
Faecal abnormality & $9(1.4)$ & $3(0.5)$ \\
Suicide attempt & $6(0.9)$ & $7(1.2)$ \\
Hyperkinesia (restless legs) & $6(0.9)$ & $4(0.7)$ \\
Micturition disorder & $5(0.8)$ & $8(1.3)$ \\
\hline
\end{tabular}

other patients no relationship with the use of SSRI was reported (for two patients the relationship was stated as 'unknown').

In Table 4, proportions of the most frequently reported adverse events in users of sertraline are shown (number of events divided by the total number of events). These results were compared with the calculated proportions of the PEM study. ${ }^{12}$ Table 5 gives an overview of the unlabelled adverse events as reported by at least $1 \%$ of the patients, specified for patients using sertraline and patients using other SSRIs. A total of 134 different unlabelled adverse events were reported by 121 patients $(9.7 \%)$. A total of 10 unlabelled events were reported by $1 \%$ or more of all users including dyspepsia, increased appetite, yawning, paraesthesia, abnormal dreaming, personality disorder (attitude change), faecal abnormalities, suicide attempt, hyperkinesia and micturition disorder.

\section{DISCUSSION}

In this observational study we evaluated the safety profile of sertraline compared to other SSRIs just after the introduction of sertraline onto the Dutch market. Observational, postmarketing research may offer more insights into the safety of a drug in actual daily practice compared to (pre-registration) trials. However, reporting bias plays an important role in these types of study. Experience with SSRIs that have been available for a longer period of time may result in an underreporting of AEs and therefore in a relative overestimation of AEs in sertraline which had been on the market for a shorter time. Furthermore, we used open questions in the patient report forms (i.e. no standardized checklist) which allows for a more spontaneous collection of adverse events. Close monitoring of completed forms and an emphasis on completion of forms at each 
follow-up visit ensured that all AEs reported by the patients were collected. Nevertheless, underreporting may still have occurred, however we have no reason to believe that underreporting was different for sertraline compared to the other SSRIs. Possible limitations may also lie in the selection of psychiatrists or patients. However, we have no reason to believe that reporting of adverse events was different between the psychiatrists or groups of patients, making the results only applicable for this population. ${ }^{13}$

Safety of SSRIs in general and of sertraline in particular has been evaluated in studies with several designs. In RCTs, comparable safety profiles of SSRIs were found in general, including gastrointestinal effects, headache and agitation, anxiety or insomnia. ${ }^{14-18}$ We also found nausea and diarrhoea and headache to be the most frequently reported AEs in users of sertraline. However, in case reports specific adverse drug reactions in individual patients are described including galactorrhoea with paroxetine use, ${ }^{19}$ extrapyramidal effects, panic attacks, hyponatremia, akathisia, seizures, galactorrhoea, mania, stuttering, hypothyroidism, hair loss and agranulocytosis with sertraline use, ${ }^{20}$ cardiovascular effects with fluoxetine use $\mathrm{e}^{21}$ and bleeding with sertraline, paroxetine and fluoxetine. ${ }^{22}$ Of these, mania, hyponatremia, hair loss and bleeding were also reported in our study although in a small minority of patients. Panic attacks or agitation was seen in a considerable number of patients with no significant difference between sertraline and other SSRIs. We found that a total of 15 patients $(1.2 \%)$ were hospitalized due to suicide attempts. Patients using other SSRIs were more often hospitalized for suicide attempts compared to patients using sertraline. Our data is insufficient to evaluate the underlying mechanisms of antidepressant therapy which may have led to suicide as proposed by Healy et $a l^{23}$ We found lower figures of suicide (attempts) than other epidemiological studies referred to by Healy et al., possibly because we set up our study in psychiatric practice and we only evaluated patients using SSRIs.

Our results were comparable to data on SSRIrelated adverse effects from a spontaneous reporting system in Sweden. ${ }^{24}$ In that study a variety of adverse reactions were reported, neurological symptoms, psychiatric and gastrointestinal symptoms being the most frequent. In users of sertraline more psychiatric symptoms were found compared to other SSRIs. Gastrointestinal symptoms were more often reported for fluvoxamine and dermatological symptoms were more often reported with fluoxetine. The authors also reported a high frequency of unlabelled AEs.
Mackay et al. have described the safety profiles of four SSRIs (fluvoxamine, fluoxetine, sertraline and paroxetine) in an observational cohort study using the British Prescription Event Monitoring (PEM) system. ${ }^{11}$ The authors compared AEs in cohorts of more than 10000 patients per SSRI (fluoxetine, fluvoxamine, paroxetine or sertraline) as reported by general practitioners (GPs). Nausea, vomiting, and malaise were the most frequently reported AEs for all SSRIs. Fluvoxamine was associated with a higher overall incidence of AEs compared to the other SSRIs. The side-effect profiles of the four SSRIs studied were comparable for frequently reported events. Nausea/ vomiting, headache/migraine, diarrhoea, dizziness, insomnia and tremor were also reported by Mackay et al. to be among the 10 most frequently reported adverse events in patients using sertraline. ${ }^{11}$

We found higher frequencies for autonomic events but lower frequencies for gastrointestinal events. Mackay et al. also reported between-drug differences that they interpreted as 'important' for some less frequently reported AEs.

Differences in outcomes in observational studies may be caused by differences in prescriber, indications, doses, duration of use, patient characteristics, publicity, year of introduction and different patterns of drugs. ${ }^{6,26}$ Two main differences between our study and the PEM study are the setting and the means of collecting data. Our data were derived from psychiatric practice while in the PEM studies the prescriber population consisted of GPs. Psychiatrists may differ from GPs in their judgements about AEs and their reporting thereof. Furthermore, at every patient visit the psychiatrists filled in a report form in which adverse events were mentioned by means of an open question (no checklist). This manoeuvre may result in a data collection more similar to an RCT than to the results of GPs' reporting in the PEM study.

In this observational study with open questions we found over 100 different unlabelled AEs. This finding is consistent with results of other studies using spontaneous reporting systems and gives insight into rare or unlabelled AEs which are frequently not mentioned in randomized clinical trials or standardized lists. ${ }^{24,27,28}$ However, causality with use of an SSRI remains unclear and other potential risk factors including age, sex, high dosages, concomitant comorbidity and comedication makes interpretation of these studies difficult. Egberts $e t$ al. found that patients reported labelled and unlabelled AEs earlier than professionals did. ${ }^{29} \mathrm{In}$ the design of this study patients were asked by the psychiatrists to report all events irrespective of the possible association with the use of an SSRI to avoid the 
psychiatrist's perception of known adverse drug reactions. We did not include concomitant co-medication in our analysis, which could have been related to a number of (unlabelled) AEs.

Channelling of newly marketed drugs into patient populations with pre-existing safety problems may confound the results of an observational study. Previously we have reported that channelling of sertraline was absent in early prescriptions of the drug after its market launch in the 1990s. ${ }^{13}$ Furthermore, the results of this study indicate no large differences in safety between a new SSRI (sertraline) and the SSRIs which had already been on the market in The Netherlands for years. We found no channelling effect, although papers by Petri and Egberts reported channelling patterns when comparing 'new' and 'old' antidepressants. ${ }^{4,5}$ Apparently, channelling is highly prevalent when there is a change from an 'old class' to a 'new class' of drugs, as was the case when the SSRIs replaced the tricyclic antidepressants (TCAs). Once the 'new class' is established new compounds in that class find their way onto the market in a much smoother fashion than when there is a between-class transition. This is probably true for SSRIs, a therapeutic category with relatively high rates of efficacy and tolerability. However, channelling studies in the field of nonsteroidal anti-inflammatory drugs (NSAIDs) where ample concern about drug-induced gastropathy exists, show that even in a market with dozens of similar existing compounds, each new introduction means the hope and promise of fewer safety problems, subsequently leading to selective prescribing of these compounds to vulnerable patients.

\section{KEY POINTS}

- Safety of SSRIs in clinical practice should be evaluated in the context of usage patterns and the dynamics of the prescribing of new drugs in often overcrowded markets of existing compounds with similar therapeutic characteristics

- The most frequently reported AEs were nausea, headache, diarrhoea, sweating and dizziness. Users of sertraline reported significantly more diarrhoea and abdominal pain compared to users of other SSRIs, but the overall safety profile of sertraline was comparable to that in other studies

- Almost three out of four patients reported an adverse event. Over 100 different unlabelled adverse events were reported
In conclusion, we found that the most frequently reported AEs were nausea, headache, diarrhoea, sweating and dizziness. Users of sertraline significantly more often reported diarrhoea and abdominal pain compared to users of other SSRIs, but the overall safety profile of sertraline was comparable with that reported in other studies. Also, we found over 100 unlabelled AEs, mostly assessed as 'mild'. Although channelling of a newly introduced drug may have occurred, in this study we found no differential safety profile of sertraline.

\section{ACKNOWLEDGEMENTS}

We thank Willem A. Nolen $\mathrm{PhD}$, for his valuable comments in preparing this manuscript.

We thank M. Gerrits (The Netherlands Pharmacovigilance Foundation) for his contribution in explaining and assisting with the WHO coding system.

This study received unconditional grant support from Pfizer bv.

\section{REFERENCES}

1. Meyboom RH, Egberts AC, Gribnau FW, Hekster YA. Pharmacovigilance in perspective. Drug Safe 1999; 21: 429-447.

2. Meyboom RH, Hekster YA, Egberts AC, Gribnau FW, Edwards IR. Causal or casual? The role of causality assessment in pharmacovigilance. Drug Safe 1997; 17: 374-389.

3. Strom BL. Overview of different logistical approaches to postmarketing surveillance. J Rheumatol 1988; 17(Suppl.): 9-13.

4. Egberts AC, Lenderink AW, de Koning FH, Leufkens HG. Channeling of three newly introduced antidepressants to patients not responding satisfactorily to previous treatment. J Clin Psychopharmacol 1997; 17: 149-155.

5. Petri H, Heerdink E, Leufkens H. Channeling of antidepressant drugs to patients with cardiovascular disease. Post Marketing Surveil 1991; 5: 159-167.

6. Leufkens H, Urquhart J. Variability in patterns of drug usage. J Pharm Pharmacol 1994; 46(Suppl. 1): 433-437.

7. Medicines Control Agency, Committee on Safety of Medicines, Royal College of General Practitioners, British Medical Association of Pharmaceutical Industry. Guidelines for Company-sponsored Safety Assessment of Marketed Medicines (SAMM). Br J Clin Pharmacol 1994; 38: 95-97.

8. Anonymous. Causality Assessment of Suspected Adverse Drug Reactions. WHO Collaborating Centre for International Drug Monitoring: Uppsala, 1991.

9. Anonymous. WHO Adverse Drug Reaction Dictionary. WHO Center for International Drug Monitoring: Uppsala, 1995.

10. Anonymous. Definitions and Standards for Expedited Reporting. ICH Harmonised Tripartite Guideline. Recommended for adoption at step 4 of the ICH Process on 27 October 1994 by the ICH Sterring Committee, 1994.

11. Mackay F, Dunn N, Wilton L, Pearce G, Freemantle S, Mann R. A comparison of fluvoxamine, fluoxetine, sertraline and paroxetine examined by observational cohort studies. Pharmacoepidemiol Drug Safe 1997; 6: 235-246. 
12. Freemantle S, Pearce G, Wilton L, Mackay F, Mann R. The incidence of the most commonly reported events with 40 newly marketed drugs - A study by Prescription Event Monitoring. Pharmacoepidemiol Drug Safe 1997; 6(Suppl. 1): 1-8.

13. Meijer W, Heerdink E, Pepplinkhuizen L, Eijk JV, Leufkens H. Prescribing patterns in patients using new antidepressants. Br J Clin Pharmacol 2001; 51: 181-183.

14. Tollefson GD. Adverse drug reactions/interactions in maintenance therapy. J Clin Psychiatry 1993; 54: 48-58.

15. Tollefson GD, Holman SL, Sayler ME, Potvin JH. Fluoxetine, placebo, and tricyclic antidepressants in major depression with and without anxious features. J Clin Psychiatry 1994; 55: 50-59.

16. Zanardi R, Franchini L, Gasperini M, Perez J, Smeraldi E. Double-blind controlled trial of sertraline versus paroxetine in the treatment of delusional depression. Am J Psychiatry 1996; 153: 1631-1633.

17. Tignol J. A double-blind, randomized, fluoxetine-controlled, multicenter study of paroxetine in the treatment of depression. J Clin Psychopharmacol 1993; 13(Suppl. 2): S18-S22.

18. Rapaport M, Coccaro E, Sheline Y, et al. A comparison of fluvoxamine and fluoxetine in the treatment of major depression. J Clin Psychopharmacol 1996; 16: 373-378.

19. Bonin B, Vandel P, Sechter D, Bizouard P. Paroxetine and galactorrhea. Pharmacopsychiatry 1997; 30: 133-134.

20. Lambert MT, Trutia C, Petty F. Extrapyramidal adverse effects associated with sertraline. Prog Neuropsychopharmacol Biol Psychiatry 1998; 22: 741-748.

21. Pacher P, Ungvari Z, Kecskemeti V, Furst S. Review of cardiovascular effects of fluoxetine-A selective serotonin reuptake inhibitor, compared to tricyclic antidepressants. Curr Med Chem 1998; 5: 381-390.

22. Lake MB, Birmaher B, Wassick S, Mathos K, Yelovich AK. Bleeding and selective serotonin reuptake inhibitors in childhood and adolescence. J Child Adolesc Psychopharmacol 2000; 10: 35-38.

23. Healy D, Langmaak C, Savage M. Suicide in the course of the treatment of depression. J Psychopharmacol 1999; 13: 94-99.

24. Spigset O. Adverse reactions of selective serotonin reuptake inhibitors: reports from a spontaneous reporting system. Drug Safe 1999; 20: 277-287.

25. Mann R, Wilton L, Pearce G, Mackay F, Dunn N. PrescriptionEvent Monitoring (PEM) in 1996-A method of noninterventional observational cohort pharmacovigilance. Pharmacoepidemiol Drug Safe 1997; 6(Suppl. 1): 1-52.

26. Leufkens H, Heerdink E. (Post-marketing surveillance (letter)). Ned Tijdschr Geneeskd 1996; 140: 1201-1202.

27. Alvarez-Requejo A, Carvajal A, Begaud B, Moride Y, Vega T, Arias LH. Under-reporting of adverse drug reactions. Estimate based on a spontaneous reporting scheme and a sentinel system. Eur J Clin Pharmacol 1998; 54: 483-488.

28. Martin RM, Kapoor KV, Wilton LV, Mann RD. Underreporting of suspected adverse drug reactions to newly marketed ('black triangle') drugs in general practice: observational study. BMJ 1998; 317: 119-120.

29. Egberts T, Smulders M, Koning DF, Meyboom R, Leufkens H. Can adverse drug reactions be detected earlier? A comparison of reports by patients and professionals. BMJ 1996; 313: $530-531$. 